\title{
Competition between phonon superconductivity and Kondo screening in mixed valence and heavy fermion compounds.
}

\author{
Victor Barzykin \\ Department of Physics and Astronomy, \\ University of Tennessee, Knoxville, TN 37996-1200 \\ L. P. Gor'kor* \\ National High Magnetic Field Laboratory, Florida State University, \\ 1800 E. Paul Dirac Dr., Tallahassee, Florida 32310
}




\begin{abstract}
We consider competition of Kondo effect and s-wave superconductivity in heavy fermion and mixed valence superconductors, using the phenomenological approach for the periodic Anderson model. Similar to the well known results for single-impurity Kondo effect in superconductors, we have found principal possibility of a re-entrant regime of the superconducting transition temperature, $T_{c}$, in heavy fermion superconductors in a narrow range of model parameters and concentration of f-electrons. Suppression of $T_{c}$ in mixed valence superconductors is much weaker. Our theory has most validity in the low-temperature Fermi liquid regime, without re-entrant behavior of $T_{c}$. To check its applicability, we performed the fit for the $x$-dependence of $T_{c}$ in $\mathrm{Ce}_{1-x} \mathrm{La}_{x} \mathrm{Ru}_{3} \mathrm{Si}_{2}$ and obtained an excellent agreement with the experimental data, although no re-entrance was found in this case. Other experimental data are discussed in the light of our theoretical analysis. In particular, we compare temperatures of the superconducting transition for some known homologs, i.e., the analog periodic lattice compounds with and without f-elements. For a few pairs of homologs superconductivity exists only in the heavy fermion materials, thus confirming uniqueness of superconductivity mechanisms for the latter. We suggest that for some other compounds the value of $T_{c}$ may remain of the same order in the two homologs, if superconductivity originates mainly on some light Fermi surface, but induces sizable superconducting gap on another Fermi surface, for which hybridization or other heavy fermion effects are more significant. By passing, we cite the old results that show that the jump in the specific heat at the transition reflects heaviness of carriers on this Fermi surface independently of mechanisms responsible for superconductivity.
\end{abstract}

PACS numbers: 74.70.Tx, 74.62.-c, 74.20.-z, 74.20.Fg

*Also at L.D. Landau Institute for Theoretical Physics, Chernogolovka, 142432, Russia 


\section{INTRODUCTION}

Since the discovery of the first heavy fermion (HF) system, $\mathrm{CeAl}_{3}$, in 1975 1], these compounds have attracted enormous theoretical and experimental interest due to their fascinating properties. The most striking feature of these systems, their extremely large effective mass $m^{*} \sim(100-1000) m_{e}$ of charge carriers, is qualitatively understood in terms of unification of magnetic degrees of freedom with the ones of itinerant electrons. The magnetic and superconducting properties of these compounds are both rich and puzzling (For a review, see $[2,3])$. Unconventional superconducting properties may be independent of mechanism, and related to the unusual symmetry of the order parameter (for reviews, see Refs. [4, 5] ). However, unlike common superconductors, the microscopic mechanisms of superconductivity (SC) in these materials for the most part remain unknown. At least a partial answer to it could be in the question whether conventional, i.e., phonon-mediated superconductivity [6] is excluded for them. In what follows, we address this issue.

The theoretical framework for studying heavy fermion metals is the periodic Anderson model, considered below in section II. Section III sums up the results of the phenomenological approach. In sections IV and V the main theoretical formulas for s-wave superconductivity are derived in the same frameworks. In section VI we discuss some scarce experimental data to show that, although there are cases where new mechanisms seem to be necessary, in many other cases phonon-mediated exchange may remain as the cause of superconductivity. In section VII we draw our conclusions.

The single site Anderson impurity problem in a normal metal has been solved[2, 7]. However, even for the low impurity concentration, the competition of superconductivity and Kondo effect or mixed valency remains a problem, for which an exact answer was not obtained theoretically. It is well known that the pair-breaking action of scattering on magnetic impurities in s-wave superconductors leads to a drastic suppression of the transition temperature with increasing impurity concentration. Taking the Kondo screening into account, however, this answer is less obvious, since at low temperatures the Kondo singlet state acts as a non-magnetic impurity [2], and thus a finite concentration of such impurities may not significantly change $T_{c}$. Competition of superconductivity and Kondo effect in alloys has been studied using various approximate methods, starting from the pioneering work of Müller-Hartmann and Zittartz [8], and we summarize the results below 
briefly.

We stress, however, that our main interest lies in the study of superconductivity of dense systems, especially of stoichiometric heavy fermion compounds, where at low temperatures the Fermi liquid regime becomes restored, and theoretical methods based on impurity scattering lose ground. We shall try to make use of the fact that many Ce- and U-based compounds have their homologs, i. e., stoichiometric compounds with non-magnetic elements like Y, La, Lu, substituting the rare-earth or actinide elements. For a number of them continuous alloy composition range is available to trace whether the superconducting transition temperature varies drastically from a phonon-like $T_{c}$ for non-magnetic compounds to a new mechanism in $\mathrm{HF}$ - or mixed valence (MV) compounds.

\section{LIMITING REGIMES IN CONCENTRATION AND MODEL PARAMETERS.}

Müller-Hartmann and Zittartz [8] used Nagaoka decoupling scheme for the Green's functions. The Nagaoka approximation fails at temperatures below the Kondo temperature, yielding non-analytic features in all physical properties. Thus, this scheme is expected to fail in the Fermi liquid regime, when the superconducting transition temperature is much less than the Kondo temperature. Nevertheless, this theory[8] has been successfully applied to many Kondo alloys, such as (La, Ce) $\mathrm{Al}_{2}$, (La,Th)Ce, and other Ce compounds.

The main result [8] is that, instead of the usual paramagnetic pair breaking curve 9], the dependence of $T_{c}$ on concentration acquires, due to the Kondo screening, a characteristic "S"shape with a re-entrant behavior. However, $T_{c}$ never goes to zero at low temperatures. Such re-entrant behavior of $T_{c}$ has been observed in the heavy fermion alloys (La,Ce) $\mathrm{Al}_{2}[10,11]$, (La,Th)Ce[12], and (La,Y)Ce[13, 14]. In particular, three transition temperatures were

clearly seen in $\mathrm{La}_{.7915} \mathrm{Ce}_{0.0085} \mathrm{Y}_{.20}[13]$. As the temperature was lowered, the first transition $T_{c 1}$ (from normal to superconducting state) was observed at $0.55 \mathrm{~K}$, the second transition $T_{c 2}$ (from superconducting to normal state) at $0.27 \mathrm{~K}$, and the third transition (back to superconducting state) at 0.05K. Significant deviations from the Abrikosov-Gor'kov theory were also observed in Kondo superconductors LaCe and LaGd[15], and PbCe and InCe films [16]. 
In its simplest form, the single-impurity Anderson Hamiltonian has the following form:

$$
\begin{aligned}
H_{\text {single }}= & \sum_{\boldsymbol{k} \sigma} \epsilon_{k} c_{\boldsymbol{k} \sigma}^{\dagger} c_{\boldsymbol{k} \sigma}+\sum_{\sigma} E_{0} f_{\sigma}^{\dagger} f_{\sigma} \\
& +V \sum_{\boldsymbol{k} \sigma}\left(c_{\boldsymbol{k} \sigma}^{\dagger} f_{\sigma}+H . c .\right)+U n_{\uparrow} n_{\downarrow},
\end{aligned}
$$

where $E_{0}$ is the energy of a localized orbital, $U$ is the on-site Coulomb repulsion energy, and $V$ is the hybridization integral between localized states and conduction states $\epsilon_{k}$. The broadening of the local level due to hybridization is given by the golden rule:

$$
\Gamma=\pi \rho V^{2},
$$

where $\rho$ is the single-spin density of states at the Fermi energy. The possible $f$-configurations for $C e$ and $U$ are $f^{0}, f^{1}$, and $f^{2}$. For $\mathrm{Yb}\left(f^{12}, f^{13}, f^{14}\right)$, one can treat Eq.(1) as the Hamiltonian for holes.

The renormalization group analysis of the model Eq.(11) has been performed by Haldane [17], who has shown that there are two fixed points:

(1) The Kondo regime,

$$
-\epsilon_{f} \gg \Gamma,
$$

in which the renormalized impurity level $\epsilon_{f}$ stops well below the chemical potential and at very large $U>0$ is indistinguishable from a local spin. Charge fluctuations on the site are negligible, while the local spin interacts antiferromagnetically with spins of conduction electrons via exchange coupling $J=V^{2} / \epsilon_{f}<0$. At high temperatures local spins behave as pair-breaking paramagnetic centers $[9,18]$. Below a characteristic temperature, $T_{K}$, the local moments become screened, and the Fermi liquid regime sets in [2]. For Ce, it corresponds to the $f^{1}$ configuration.

(2) The mixed valence regime,

$$
\left|\epsilon_{f}\right| \sim \Gamma .
$$

In this regime the two configurations, say, $f^{0}$ and $f^{1}$ for Ce, are approximately degenerate. The system is characterized by a time scale for spin fluctuations (charge fluctuations are strong as well).

If the f-level is taken above the chemical potential, and the hybridization, $V$, is weak:

$$
E_{0} \simeq \epsilon_{f} \gg \Gamma,
$$


then, the impurity is mostly "empty", so that the scattering is mostly nonmagnetic in character. Nevertheless, finite hybridization of electrons with correlated impurity levels introduces an effective repulsion between conduction electrons with opposite spin, which grows with increased concentration of impurities, and causes pair weakening. In the Hartree-Fock approximation such pair weakening caused by resonant impurity scattering has been studied by Kaiser [19], Shiba 20], and Schlottmann [21]. This results in a modified exponential decay of $T_{c}$ with increased impurity concentration [19]. The Hartree-Fock approach is only valid for small enough Coulomb repulsion[21], $U / \Gamma \ll 1$. Nevertheless, it has produced a good description of $T h C e$ 22] and some lanthanide alloys with large Kondo or spin fluctuation scales.

The presence of Kondo effect significantly complicates the treatment of $T_{c}$ suppression. The approach [8] that uses the Nagaoka decoupling only works well for small values of the Kondo scale $T_{K}<T_{c 0}$. In the Fermi liquid regime, the theory was developed by Matsuura, Ichinose, and Nagaoka 23], and by Sakurai[24]. For the Fermi liquid fixed point, pair weakening occurs through virtual polarization of the Kondo ground state [23, 24]. This theory is valid when $T_{K} \gg T_{c 0}$. The behavior of magnetic impurities in strongly coupled superconductors has been studied numerically [25] and analytically [26], with the result that strong coupling weakens the effect of Kondo impurities by a factor $1+\lambda$, where $\lambda$ is due to electron-phonon interaction. The low-temperature regime has also been studied in the slave boson $1 / N$ formalism[27]. A unified treatment of superconductivity in presence of Anderson impurities in the NCA approximation $\left(T_{c} \gg T_{K}\right)$ has been done by Bickers and Zwicknagl[28].

Analysis of superconducting properties of alloys in this regime has been done perturbatively by Schlottmann[29]: $T_{c}$ decreases at first linearly with concentration, then exponentially. In addition, this regime was studied at zero temperatures [30] using the largedegeneracy expansion of Gunnarsson and Schönhammer [31]. For the periodic lattice, superconductivity in a mixed valence compound was analyzed using the Green function approach[32]. Experimentally, superconductivity in mixed valence regime has been studied in detail for $\mathrm{CeRu}_{3} \mathrm{Si}_{2}[33], \mathrm{CeRu}_{2}$, and $\mathrm{CeIr}_{3}[34]$.

As it was mentioned in introduction, below we study the competition of superconductivity and Kondo effect in concentrated alloys and Anderson lattices at low temperatures. The influence of impurities on the superconducting state in heavy fermions has also attracted 
some experimental interest. For example, superconductivity in presence of non-magnetic impurities has been studied in heavy fermion superconductor $\mathrm{CeCu}_{2} \mathrm{Si}_{2}$ [35, 36]. The peculiar properties of $\mathrm{U}_{1-x} \mathrm{Th}_{x} \mathrm{Be}_{13}$ are also well known[37, 38]. Numerous experiments have been done in other alloys, such as $\mathrm{Th}_{1-x} \mathrm{Ce}_{x}, \mathrm{Th}_{1-x} \mathrm{U}_{x}, \mathrm{Al}_{1-x} \mathrm{Mn}_{x}, \mathrm{La}_{3-x} \mathrm{Ce}_{x} \mathrm{In}$. We refer the reader to Ref. 39] for a detailed review of relevant experiments. However, the theoretical model developed below leaves scattering effects aside. We apply our results only to systems where the latter does not play important role, because the f-electrons go into bands (i.e., where no sharp decrease of $T_{c}$ at low concentrations was observed). For the heavy fermion systems the common assumption since Ref. [40] is that SC forms on a heavy Fermi surface due to electron-electron interactions, and then induces SC on other parts (see, e.g., in Ref. 41]). To the contrary, we begin with the phonon mechanism.

\section{HEAVY FERMION LIQUID AND RENORMALIZED BANDS.}

As usual, we start the consideration of a heavy fermion liquid by writing the periodic Anderson model,

$$
H=H_{0}+H_{V}+H_{e f}
$$

where

$$
H_{0}=\sum_{\boldsymbol{k} \sigma} \epsilon_{k} c_{\boldsymbol{k} \sigma}^{\dagger} c_{\boldsymbol{k} \sigma}+\sum_{i \sigma} E_{0} f_{i \sigma}^{\dagger} f_{i \sigma}+\sum_{i} U f_{i \uparrow}^{\dagger} f_{i \downarrow}^{\dagger} f_{i \downarrow} f_{i \uparrow}
$$

Here the creation and annihilation operators for the f-electrons, $f_{i \sigma}^{\dagger}$ and $f_{i \sigma}$, carry the site index $i$, and there is a Coulomb interaction at each site for the f-electrons. The operators $c_{\boldsymbol{k} \sigma}^{\dagger}$ and $c_{\boldsymbol{k} \sigma}$ correspond to delocalized Bloch states. The hybridization term in the model Hamiltonian, $H_{V}$, accounts for the $s-d$ hybridization between the f-electrons and the Bloch states:

$$
H_{V}=\sum_{i, \boldsymbol{k}, \sigma}\left(V_{\boldsymbol{k}} e^{i \boldsymbol{k} \cdot \boldsymbol{R}_{i}} f_{i \sigma}^{\dagger} c_{\boldsymbol{k} \sigma}+V_{\boldsymbol{k}}^{*} e^{-i \boldsymbol{k} \cdot \boldsymbol{R}_{i}} c_{\boldsymbol{k} \sigma}^{\dagger} f_{i \sigma}\right) .
$$

Finally, the third term in the Hamiltonian Eq.(6) corresponds to the attraction, caused by the electron-phonon interaction, which we consider in the weak coupling limit here:

$$
H_{e f}=\frac{\lambda}{2} \int \Psi_{\sigma}^{\dagger}(\boldsymbol{r}) \Psi_{\sigma^{\prime}}^{\dagger}(\boldsymbol{r}) \Psi_{\sigma^{\prime}}(\boldsymbol{r}) \Psi_{\sigma}(\boldsymbol{r}) d \boldsymbol{r}
$$


where the $\Psi_{\sigma}(\boldsymbol{r})$ and $\Psi_{\sigma}^{\dagger}(\boldsymbol{r})$ are operators, which correspond to the itinerant band:

$$
\begin{aligned}
& \Psi_{\sigma}(\boldsymbol{r})=\frac{1}{(2 \pi)^{3}} \int e^{i \boldsymbol{k} \cdot \boldsymbol{r}} c_{\boldsymbol{k} \sigma} d \boldsymbol{k}, \\
& \Psi_{\sigma}^{\dagger}(\boldsymbol{r})=\frac{1}{(2 \pi)^{3}} \int e^{-i \boldsymbol{k} \cdot \boldsymbol{r}} c_{\boldsymbol{k} \sigma}^{\dagger} d \boldsymbol{k} .
\end{aligned}
$$

The on-site Coulomb repulsion $U$ is usually very large for f-electron materials, and it will be taken infinite below. To account for this, the creation/annihilation operators for $f$ electrons in $H_{V}$ would have to be taken with projection operators, which project out doubly occupied $f$-electron states [42]. A convenient way to rewrite this Hamiltonian, invented by Coleman[43], Read and Newns 44], and Barnes [45], is to introduce a new slave boson field $b_{i}^{+}$, which creates a hole on site $i$, and to rewrite the Anderson Hamiltonian in a way, which allows a $1 / N$ expansion in the number of orbitals. However, in what follows, we resort to a more phenomenological approach of Edwards [4] and Fulde[48] (see also Ref.(2])).

Introducing the set of the fermion Green's functions (for imaginary time $\tau$ ),

$$
\begin{aligned}
G_{c c}^{m}(\boldsymbol{k}, \tau) & \equiv-\left\langle T_{\tau} c_{\boldsymbol{k} m}(\tau) c_{\boldsymbol{k} m}^{\dagger}(0)\right\rangle, \\
G_{f c}^{m}(\boldsymbol{k}, \tau) & \equiv-\left\langle T_{\tau} f_{\boldsymbol{k} m}(\tau) c_{\boldsymbol{k} m}^{\dagger}(0)\right\rangle, \\
G_{f f}^{m}(\boldsymbol{k}, \tau) & \equiv-\left\langle T_{\tau} f_{\boldsymbol{k} m}(\tau) f_{\boldsymbol{k} m}^{\dagger}(0)\right\rangle,
\end{aligned}
$$

and transforming to Matsubara frequencies, we find for them the diagrammatic expansion in powers of $U$ and $V_{\boldsymbol{k}}$ for the conduction and $f$-electron Green's functions:

$$
\left[\begin{array}{cc}
i \omega_{n}-\epsilon_{f}+\mu-\Sigma_{\sigma}\left(\omega_{n}, \boldsymbol{k}\right) & -V_{\boldsymbol{k}} \\
-V_{\boldsymbol{k}} & i \omega_{n}-\epsilon_{k}+\mu
\end{array}\right]\left[\begin{array}{cc}
G_{\boldsymbol{k}, \sigma}^{f f}\left(\omega_{n}\right) & G_{\boldsymbol{k}, \sigma}^{c f}\left(\omega_{n}\right) \\
G_{\boldsymbol{k}, \sigma}^{f c}\left(\omega_{n}\right) & G_{\boldsymbol{k}, \sigma}^{c c}\left(\omega_{n}\right)
\end{array}\right]=\hat{I} .
$$

Assuming that $\Sigma_{\sigma}\left(\omega_{n}, \boldsymbol{k}\right)$ can be expanded near the Fermi surface, $|\boldsymbol{k}|=k_{F}$, and retaining the first order terms:

$$
\Sigma\left(\omega_{n}, \boldsymbol{k}\right) \simeq \Sigma\left(0, \boldsymbol{k}_{F}\right)+\left(\boldsymbol{k}-\boldsymbol{k}_{F}\right) \cdot \nabla \Sigma(0, \boldsymbol{k})_{k=k_{F}}+\omega_{n}\left(\frac{\partial \Sigma\left(\omega_{n}, \boldsymbol{k}_{F}\right)}{\partial \omega_{n}}\right)_{\omega_{n}=0},
$$

the Green's function can now be written in the form analogous to the non-interacting $(U=0)$ problem:

$$
G_{\boldsymbol{k}, \sigma}^{c c}\left(\omega_{n}\right)=\frac{1}{i \omega_{n}-\epsilon_{\boldsymbol{k}}+\mu-\frac{\left|\tilde{V}_{\boldsymbol{k}}\right|^{2}}{i \omega_{n}-\tilde{\epsilon}_{f k}}}
$$

where

$$
\begin{aligned}
\tilde{\epsilon}_{f \boldsymbol{k}} & =Z\left(\epsilon_{f}-\mu+\Sigma^{R}\left(0, k_{F}\right)+\left(\boldsymbol{k}-\boldsymbol{k}_{F}\right) \cdot \nabla \Sigma\left(0, k_{F}\right)\right), \\
\left|\tilde{V}_{\boldsymbol{k}}\right|^{2} & =Z\left|V_{\boldsymbol{k}}\right|^{2} .
\end{aligned}
$$


Since $\tilde{\epsilon}_{f \boldsymbol{k}}$ is only weakly $\boldsymbol{k}$-dependent, we can replace it with a constant, $\epsilon_{f}^{e f f}$. Furthermore, in what follows we also assume that $V_{\boldsymbol{k}}$ does not depend on the direction of $\boldsymbol{k}$, so that we can replace $\tilde{V}_{\boldsymbol{k}}$ by a constant, $\tilde{V}=\tilde{V}_{k_{F}}$. As usual, the quasiparticle residue $Z$ is given by

$$
Z=\frac{1}{1-\left[\frac{\partial \Sigma\left(\omega_{n}, \boldsymbol{k}_{F}\right)}{\partial \omega_{n}}\right]_{\omega_{n}=0}} .
$$

After making use of Eqs (15) and (19), the Green's functions acquire the form:

$$
\begin{aligned}
G_{c c}^{m}\left(\boldsymbol{k}, \omega_{n}\right) & =\frac{i \omega_{n}-\epsilon_{f}^{e f f}}{\left(i \omega_{n}-\epsilon_{f}^{e f f}\right)\left(i \omega_{n}-\xi_{\boldsymbol{k}}\right)-\tilde{V}^{2}}, \\
G_{f f}^{m}\left(\boldsymbol{k}, \omega_{n}\right) & =\frac{i \omega_{n}-\xi_{\boldsymbol{k}}}{\left(i \omega_{n}-\epsilon_{f}^{e f f}\right)\left(i \omega_{n}-\xi_{\boldsymbol{k}}\right)-\tilde{V}^{2}}, \\
G_{f c}^{m}\left(\boldsymbol{k}, \omega_{n}\right) & =\frac{\tilde{V}}{\left(i \omega_{n}-\epsilon_{f}^{e f f}\right)\left(i \omega_{n}-\xi_{\boldsymbol{k}}\right)-\tilde{V}^{2}} .
\end{aligned}
$$

From the poles of the Green's functions Eq.(20), the renormalized energy spectrum has the following form:

$$
\tilde{\xi}_{k 1,2}=\frac{\epsilon_{f}^{e f f}+\xi_{k}}{2} \pm \frac{1}{2} \sqrt{\left(\epsilon_{f}^{e f f}-\xi_{k}\right)^{2}+4|\tilde{V}|^{2}},
$$

where $\xi_{k} \equiv \epsilon_{k}-\mu$. In the limit $U \rightarrow \infty$ the effective f-band $\tilde{\epsilon}_{f \boldsymbol{k}}$ must lie above the Fermi surface, so that the total occupation of the f-level, $n_{f}$, is such that $0<n_{f}<1$. For the effective mass of quasiparticles, after expanding $\tilde{\xi}_{k 2}$ in the vicinity of the Fermi surface, one obtains:

$$
\frac{m^{*}}{m}=\frac{|\tilde{V}|^{2}}{\left(\epsilon_{f}^{e f f}\right)^{2}}+1 .
$$

In the Kondo limit, $\epsilon_{f}^{e f f}=T_{K}$ can be thought of as the Kondo temperature. The conservation of the total number of quasiparticles (Luttinger theorem) leads to the shift of the chemical potential, given by

$$
\tilde{\mu}=\mu+\frac{|\tilde{V}|^{2}}{\epsilon_{f}^{e f f}} .
$$

Note, however, that the parameters $\tilde{V}$ and $\epsilon_{f}^{e f f}$ are, in general, temperature-dependent. Then, their temperature dependence can be studied within a specific model, such as slave boson $1 / N$ approach. Due to the restriction of the slave boson approach to low temperatures, our results for phonon-mediated superconductivity are applicable to the case when both $\tilde{V}$ and $\epsilon_{f}^{e f f}$ are much greater than $T_{c}$, so that these parameters could be regarded as temperature-independent. Below we merely consider $\tilde{V}$ and $\epsilon_{f}^{e f f}>0$ as two free parameters of our theory. 


\section{GENERAL FORMULA FOR $T_{c}$.}

We can now evaluate the superconducting transition temperature, using the phonon attraction Hamiltonian Eq.(9), and our new energy spectrum Eq.(21). In what follows, we assume that the phonon cutoff $\omega_{D}$ in Eq.(9) is much greater than the Kondo temperature $\epsilon_{f}^{e f f}$, and the effective hybridization $|\tilde{V}|$,

$$
\omega_{D} \gg \max \left\{|\tilde{V}|, \epsilon_{f}^{e f f}\right\}
$$

Following Ref. 49], $T_{c}$ is obtained by evaluating the Cooper diagram,

$$
T_{c} \sum_{n} \int \frac{d \boldsymbol{k}}{(2 \pi)^{3}} G_{\boldsymbol{k}}^{c c}\left(\omega_{n}\right) G_{-\boldsymbol{k}}^{c c}\left(-\omega_{n}\right)=\frac{1}{|\lambda|} .
$$

As usual, to get rid of the cutoff dependence arising from the frequency summation, we have

to introduce a new energy scale $T_{c 0}$, for the superconducting temperature in absence of the f-electrons:

$$
T_{c 0}=\frac{2 \omega_{D} \gamma}{\pi} e^{-2 \pi^{2} /|\lambda| m k_{F}}
$$

and rewrite Eq.(25) as

$$
T_{c} \sum_{\left|\omega_{n}\right|<\omega_{D}} \int \frac{d \boldsymbol{k}}{(2 \pi)^{3}} G_{\boldsymbol{k}}^{c c}\left(\omega_{n}\right) G_{-\boldsymbol{k}}^{c c}\left(-\omega_{n}\right)=T_{c 0} \sum_{\left|\omega_{n}\right|<\omega_{D}} \int \frac{d \boldsymbol{k}}{(2 \pi)^{3}} G_{\boldsymbol{k}}^{0}\left(\omega_{n}\right) G_{-\boldsymbol{k}}^{0}\left(-\omega_{n}\right),
$$

where

$$
G_{\boldsymbol{k}}^{0}\left(\omega_{n}\right) \equiv \frac{1}{i \omega_{n}-\xi_{k}}
$$

(Note that we introduced the cutoff $\omega_{D}$ in the sum over $n$, while the integral over $\xi$ goes from $-\infty$ to $+\infty$.) After some simple but tedious transformations, we find that the Cooper bubble on the left-hand side of Eqs(25), (27) can be written as:

$$
\Pi\left(\omega_{n}, \boldsymbol{k}\right) \equiv G_{\boldsymbol{k}}^{c c}\left(\omega_{n}\right) G_{-\boldsymbol{k}}^{c c}\left(-\omega_{n}\right)=\frac{\omega_{n}^{2}+\left(\epsilon_{f}^{e f f}\right)^{2}}{\left(\omega_{n}^{2}+\tilde{\xi}_{k 1}^{2}\right)\left(\omega_{n}^{2}+\tilde{\xi}_{k 2}^{2}\right)},
$$

where $\tilde{\xi}_{k 1,2}$ are given by Eq.(21). Integrating Eq.(27) by $\xi_{k}$, we get:

$$
T_{c} \sum_{\left|\omega_{n}\right|<\omega_{D}} \frac{\pi\left(\omega_{n}^{2}+\left(\epsilon_{f}^{e f f}\right)^{2}\right.}{\left|\omega_{n}\right|\left(\omega_{n}^{2}+\left[\epsilon_{f}^{e f f}\right]^{2}+\tilde{V}^{2}\right)}=T_{c 0} \sum_{\left|\omega_{n}\right|<\omega_{D}} \frac{\pi}{\left|\omega_{n}\right|},
$$

which can be rewritten using the definition of the digamma function,

$$
\Psi(z) \equiv-\gamma-\sum_{n=1}^{\infty}\left(\frac{1}{z+n-1}-\frac{1}{n}\right)
$$


in the following form:

$$
\left[\frac{\left(\epsilon_{f}^{e f f}\right)^{2}}{\tilde{V}^{2}}+1\right] \ln \left[\frac{T_{c}}{T_{c 0}}\right]=\Psi\left(\frac{1}{2}\right)-\frac{1}{2} \Psi\left(\frac{1}{2}+i \frac{A}{2 \pi T_{c}}\right)-\frac{1}{2} \Psi\left(\frac{1}{2}-i \frac{A}{2 \pi T_{c}}\right),
$$

where

$$
A=\sqrt{\left(\epsilon_{f}^{e f f}\right)^{2}+\tilde{V}^{2}}=\epsilon_{f}^{e f f} \sqrt{\frac{m^{*}}{m}} .
$$

Alternatively, we can use the definition of the effective mass, Eq.(22), to write:

$$
\left[\frac{m^{*}}{m^{*}-m}\right] \ln \left[\frac{T_{c}}{T_{c 0}}\right]=\Psi\left(\frac{1}{2}\right)-\frac{1}{2} \Psi\left(\frac{1}{2}+i \frac{A}{2 \pi T_{c}}\right)-\frac{1}{2} \Psi\left(\frac{1}{2}-i \frac{A}{2 \pi T_{c}}\right),
$$

which is our main result for a stoichiometric compound.

Let us now analyze this equation in more detail. First, we note that when $\epsilon_{f}^{e f f}=0$, Eq.(34) coincides with the equation for $T_{c}$ for the paramagnetic pair breaking effect of magnetic field, if we replace $A=\mu_{B} B$. However, unlike for the paramagnetic effect, in general the factor on the left-hand side of Eq.(34) is not unity. This means that $T_{c} \neq 0$ for non-zero $\epsilon_{f}^{e f f}>0$ for any choice of parameters, even though it could become very small.

To clarify Eq.(34) more, let us consider the limiting cases. The two obvious cases are $A \gg T_{c 0}$ and $A \ll T_{c 0}$.

1) $A \ll T_{c 0}$. Then we find:

$$
\frac{\Delta T_{c}}{T_{c 0}}=-\frac{7 \zeta(3)}{4 \pi^{2} T_{c 0}^{2}} \tilde{V}^{2}=-\frac{7 \zeta(3)}{4 \pi^{2} T_{c 0}^{2}}\left(\epsilon_{f}^{e f f}\right)^{2}\left(\frac{m^{*}}{m}-1\right),
$$

where the coefficient $7 \zeta(3) / 4 \pi^{2} \simeq 0.21$

2) $A \gg T_{c 0}$ Expanding $\Psi$ at high $z$, we get

$$
\frac{m}{m^{*}-m} \ln \left[\frac{T_{c}}{T_{c 0}}\right]=\ln \left(\frac{\pi T_{c 0}}{2 \gamma A}\right) \text {. }
$$

Thus,

$$
\frac{T_{c}}{T_{c 0}}=\exp \left[\frac{m^{*}-m}{m} \ln \left(\frac{\pi T_{c 0}}{2 \gamma A}\right)\right]=\left(\frac{\pi T_{c 0}}{2 \gamma A}\right)^{\left(m^{*} / m\right)-1} .
$$

Finally, we note that Eq.(34) can be written in terms of dimensionless quantities,

$$
t \equiv \frac{T_{c}}{T_{c 0}}, \quad \tilde{A} \equiv \frac{A}{T_{c 0}} .
$$

Indeed, we can write it now as:

$$
\left[\frac{m^{*}}{m^{*}-m}\right] \ln t=\Psi\left(\frac{1}{2}\right)-\frac{1}{2} \Psi\left(\frac{1}{2}+i \frac{\tilde{A}}{2 \pi t}\right)-\frac{1}{2} \Psi\left(\frac{1}{2}-i \frac{\tilde{A}}{2 \pi t}\right) .
$$




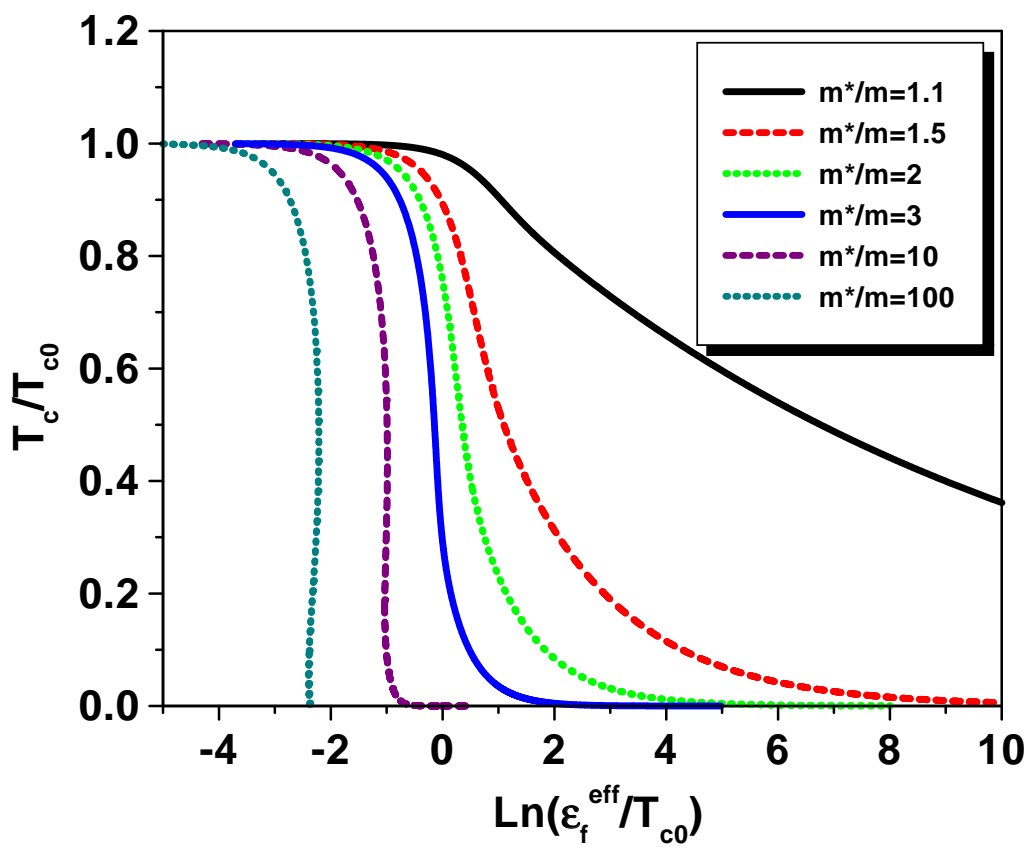

FIG. 1: Superconducting transition temperature as a function of parameters $m^{*} / m$ and $\epsilon_{f}^{e f f} / T_{c 0}$.

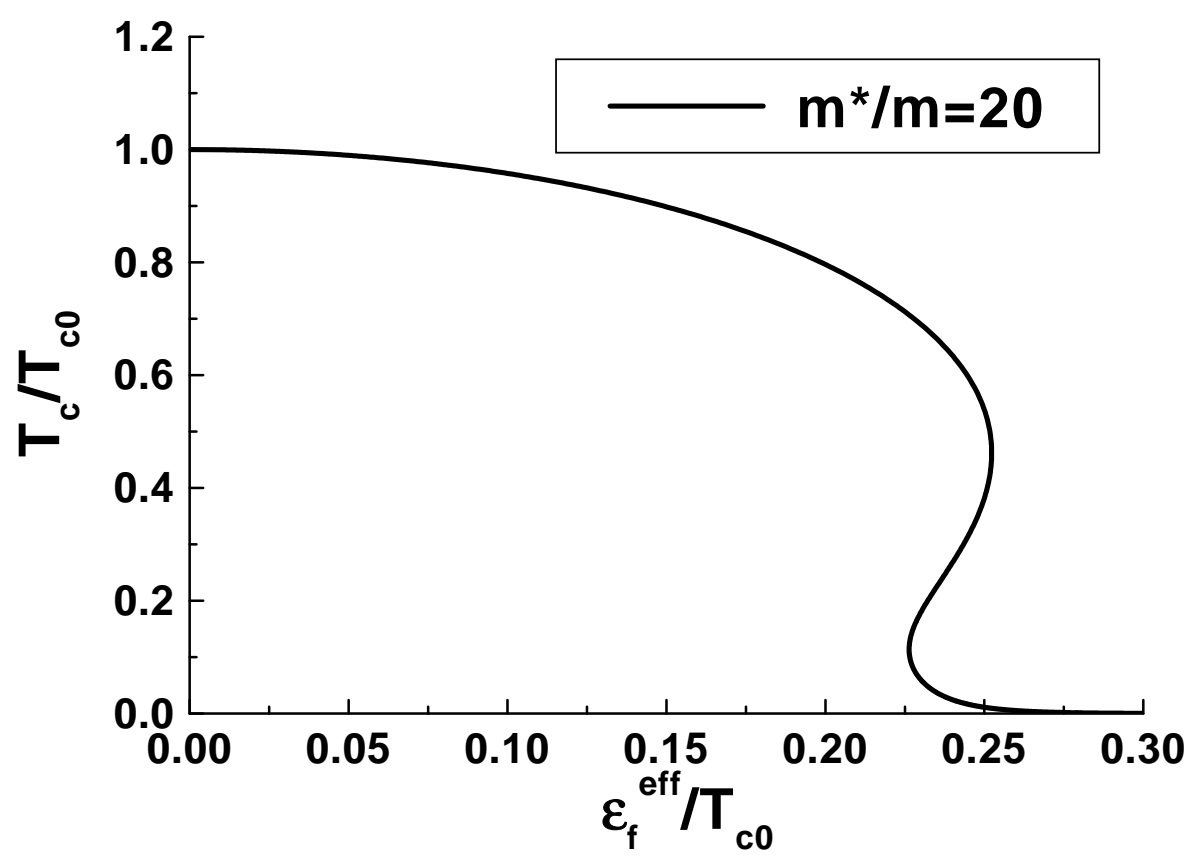

FIG. 2: Re-entrant behavior of superconducting transition temperature as a function of parameter $\epsilon_{f}^{e f f} / T_{c 0}$. 
We plot Eq.(39) as a function of two parameters, $m^{*} / m$ and $\epsilon_{f}^{\text {eff }} / T_{c 0}$, in Fig 11.

Interesting enough, the dependence of $T_{c}$ on parameters may have a characteristic S-shape re-entrance form, as shown more explicitly in Fig 2. This, and the similarity of Eqs (32), (34) to the field dependence for paramagnetic pair breaking raises two questions:

a) Is the superconducting transition always second order?

b) Is the homogeneous state the most stable state, or could a superstructure similar to Larkin-Ovchinnikov-Fulde-Ferrel (LOFF) [50, 51] state appear?

We have investigated both questions, and found that the superconducting transition is always of the second order, and that the inhomogeneous state is always energetically unfavorable. As to the S-shape dependence in Figs 1, 2, one sees that it arises at rather

specific conditions: $\epsilon_{f}^{e f f}$ must be less than $T_{c 0}$ (usually, of the order of a few $\mathrm{K}$ ). according to Eq.(22),$\tilde{V}$ is also rather weak. For a single level center it corresponds to Eq. (41) with $\Gamma \ll 1$, i.e., an almost localized level near the chemical potential that can be thermally populated. We are not aware of a material that would satisfy these criteria.

\section{SUPERCONDUCTIVITY IN DENSE ALLOYS.}

Since experiments often deal with alloys, let us consider in detail the dependence of the critical temperature on concentration in the case when $\epsilon_{f}^{e f f}$ and $m^{*} / m$ are fixed. This applies, for example, to dense mixed valence alloys in the phenomenological description of Eqs (17)-(21). For the concentration of f-elements per unit cell $x(x<1)$, after repeating the above calculations, we find for $T_{c}$ :

$$
\left[\frac{\left(\epsilon_{f}^{e f f}\right)^{2}}{x \tilde{V}^{2}}+1\right] \ln \left[\frac{T_{c}}{T_{c 0}}\right]=\Psi\left(\frac{1}{2}\right)-\frac{1}{2} \Psi\left(\frac{1}{2}+i \frac{A(x)}{2 \pi T_{c}}\right)-\frac{1}{2} \Psi\left(\frac{1}{2}-i \frac{A(x)}{2 \pi T_{c}}\right),
$$

where

$$
A(x)=\sqrt{\left(\epsilon_{f}^{e f f}\right)^{2}+x \tilde{V}^{2}} .
$$

In the derivation Eqs(15)-(17), the level $\epsilon_{f}^{e f f}$ and the effective hybridization $\tilde{V}$ correspond to the Anderson model for a single impurity, and do not change with concentration. However, we see that the concentration of f-elements enters explicitly in our new equation, with $x$ as the probability to find the $f$-level at a given site. This equation is, in fact, the same as the 
solution for the lattice problem, with $\tilde{\tilde{V}} \equiv \sqrt{x} \tilde{V}$ used as the effective hybridization. In other words, in alloys effective hybridization is "tuned up" by the concentration of $f$-levels.

At small, but finite $\epsilon_{f}^{e f f}$, i.e., in the Kondo regime, one is able, like in Fig. 2, to obtain the S-shape of $T_{c}$ - this time, however, as a function of concentration, i.e., the regime with three transition temperatures, which was the main result in Ref.[8]. Note that the approach in Ref.[8], strictly speaking, was not rigorously founded at concentrations for the re-entrant superconductivity regime. As for our Eq.(40), although it also predicts an initial decrease of $T_{c}$ at small $\mathrm{x}$ (and small $\epsilon_{f}^{e f f}$ ), as in Eq.(134), it is also deficient here, because the electron scattering on $f$-centers is not included in the framework of our derivation.

In what follows, we only discuss the case $T_{c} \ll \epsilon_{f}^{e f f}$, since it applies to most experiments on dense alloys. Then we don't expect our parameters, $\epsilon_{f}^{e f f}$ and $\tilde{V}$, to be temperaturedependent. Since $T_{c 0} \ll \epsilon_{f}^{e f f}$, we are always in the limit $A \gg T_{c 0}$. Thus, the decay of $T_{c}$ with concentration is exponential. Let us introduce, for convenience, two new dimensionless parameters, $k$ and $y$ :

$$
\begin{aligned}
& k=\frac{2 \gamma \epsilon_{f}^{e f f}}{\pi T_{c 0}}, \\
& y=\frac{m^{*}(x=1)}{m}=\frac{\tilde{V}^{2}}{\left(\epsilon_{f}^{e f f}\right)^{2}}+1 .
\end{aligned}
$$

Then we can write $T_{c}$ as a function of doping as:

$$
\frac{T_{c}}{T_{c 0}}=\exp [-x(y-1) \ln (k \sqrt{1+x(y-1)})]
$$

The doping dependence of $T_{c}$ for various choices of parameter $k$ is shown in Fig. 3, We can see that characteristic concentration where $T_{c}$ decays substantially depends mainly

on the ratio of $\epsilon_{f}^{e f f}$ and $\tilde{V}$. Thus, for Kondo impurities, where $\epsilon_{f}^{e f f} \ll \tilde{V}, T_{c}$ is rapidly suppressed, although remains non-zero at $x=1$. On the other hand, for mixed valence impurities $\epsilon_{f}^{e f f} \sim \tilde{V}$, so $T_{c}$ is suppressed exponentially, but not so steep.

\section{DISCUSSION OF THE EXPERIMENTAL DATA.}

As it would follow from our results, if a phonon (s-wave) superconductivity were present for a compound without an element with f-electrons (e.g., La or Lu), one should expect that its stoichiometric homolog with the f-electrons being present, will have a small, but finite, 


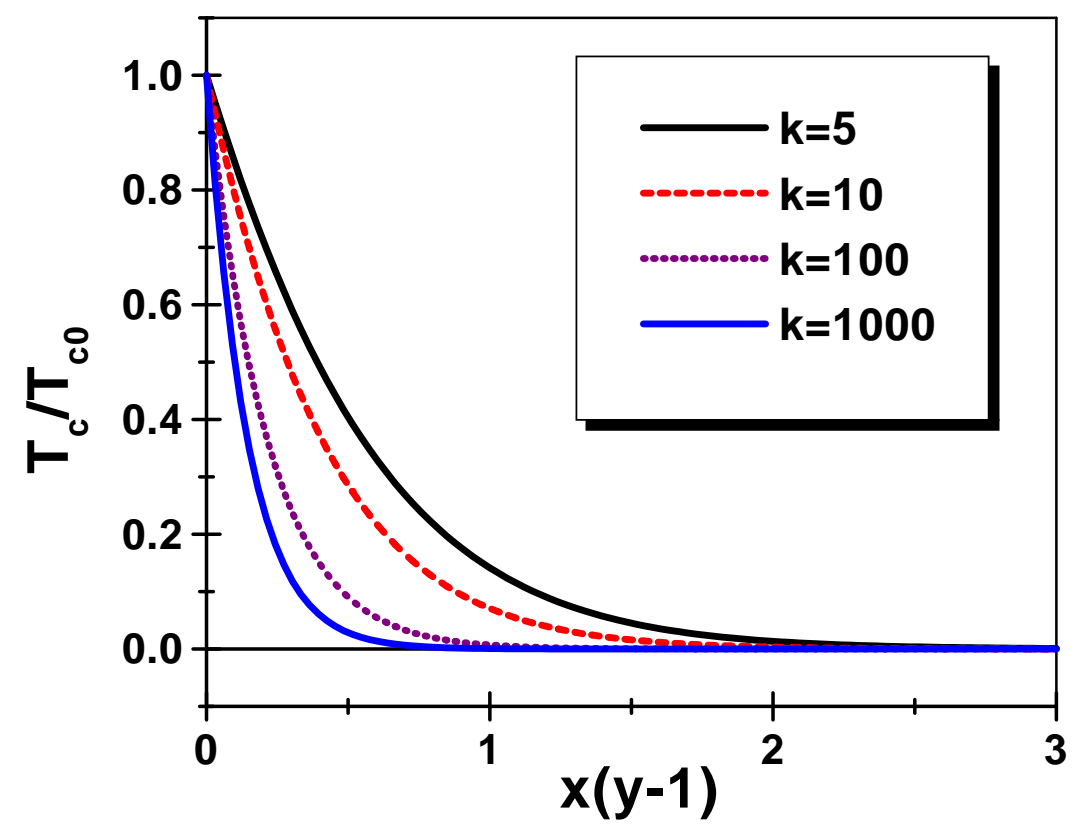

FIG. 3: Superconducting transition temperature as a function of doping $x$ in alloys.

$T_{c} \neq 0$. This prediction is, of course, not fully conclusive, for already at dilute solution of paramagnetic centers the pair-breaking scattering initially may take $T_{c}$ practically to zero, while appearing again in the periodic (dense) limit in absence of scattering. Our approach, however, is to circumvent the scattering regime, and in its framework one may estimate the phonon superconductivity $T_{c}$ at the other end through Kondo, or, more generally, the Anderson-lattice parameters of the latter. Certainly, superconductivity in a heavy fermion compound may be due to a completely different mechanism, but the analysis along the line proposed above, nevertheless, seems to be instructive. Thus, for instance, $\mathrm{LaCu}_{2} \mathrm{Si}_{2}$ is not a superconductor, while $\mathrm{CeCu}_{2} \mathrm{Si}_{2}[52$ ] is. Similarly, there are no $\mathrm{SC}$ homologs without $f$ electrons known for $\mathrm{UBe}_{13}$. This gives an additional and unambiguous argument in favor of an unconventional superconductivity mechanism for these HF materials.

Unfortunately, such decisive experimental data are rather scarce and are available mostly with the Ce-based materials. In the literature the latter are often subdivided into "strongly mixed valent" $\left(\mathrm{CeRu}_{2} ; \mathrm{CeCo}_{2}\right)$ and "weakly mixed valent" $\left(\mathrm{CeSn}_{3}, \mathrm{CePd}_{3}, \mathrm{CeBe}_{13}\right)$ compounds (see, e.g., in Ref.[33]). Among the second group no superconductors have been found, and they are usually considered to be closer to localized moments, or Kondo, ma- 


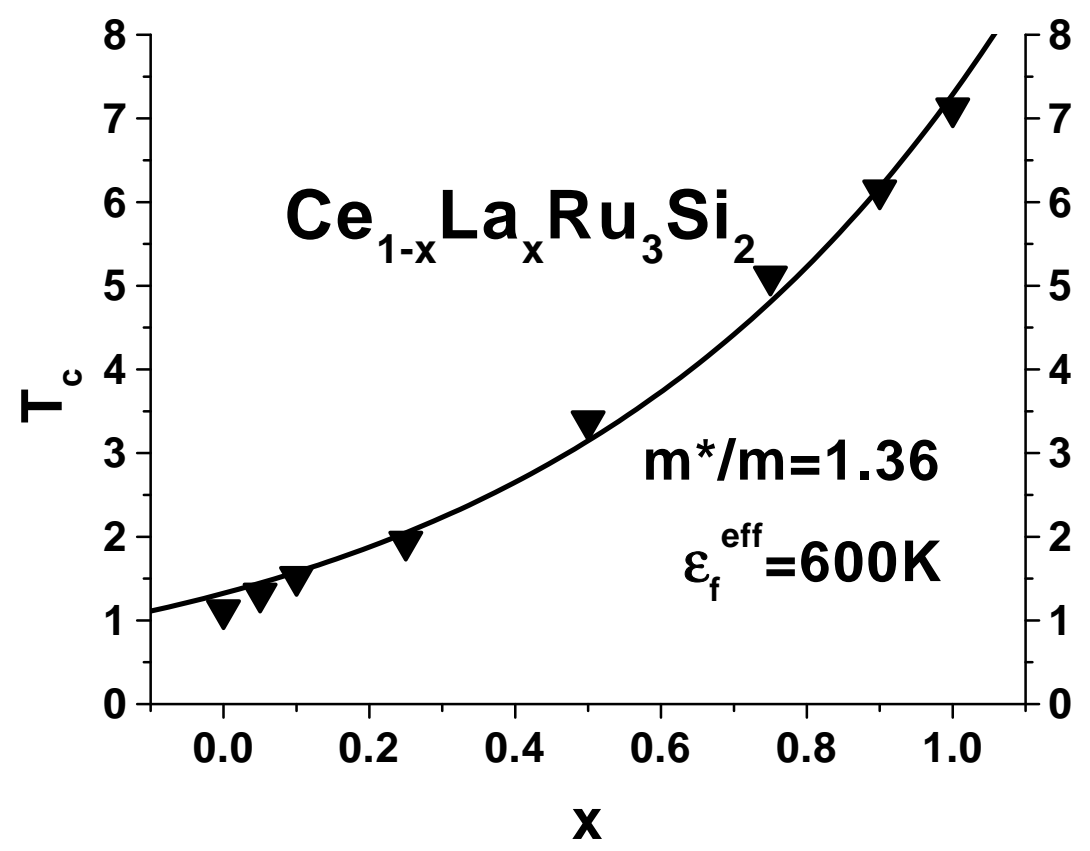

FIG. 4: Superconducting transition temperature as a function of doping $x$ in $\mathrm{Ce}_{1-x} \mathrm{La}_{x} \mathrm{Ru}_{3} \mathrm{Si}_{2}$, from Ref. [58] vs our theoretical fit.

terials. Among the first group we have pairs: $\mathrm{CeRu}_{2}\left(T_{c} \simeq 6 K\right)$ and $\mathrm{LaRu}_{2}\left(T_{c} \simeq 4 K\right)$, $\mathrm{LaPd}_{2} \mathrm{Ge}_{2}\left(T_{c} \simeq 1.1 K\right)[53]$ and $\mathrm{CePd}_{2} \mathrm{Ge}_{2}\left(T_{c} \simeq 0.07 K, P=14.6 G P a\right)[54], \mathrm{LaNi}_{2} \mathrm{Ge}_{2}$ $\left(T_{c} \simeq 0.8 K\right)[55\rfloor$ and $\mathrm{CeNi}_{2} \mathrm{Ge}_{2}\left(T_{c} \simeq 0.2 K\right)[56]$. No superconductivity has ever been found for $\mathrm{Yb}$ compounds [57]. Common view (e.g., in Ref.[34]) is that in mixed valence compounds f-electrons merely hybridize with one of conduction bands, thus supplying additional electrons into that band.

We consider in more detail $\mathrm{CeRu}_{3} \mathrm{Si}_{2}$ and its homolog $\mathrm{LaRu}_{3} \mathrm{Si}_{2}$ with its $\mathrm{Ru}$-derived $4 \mathrm{~d}$ band, where the data for the continuous alloying are available [58]. In Fig 4 we show the fit for $T_{c}$ in the $\mathrm{Ce}_{1-x} \mathrm{La}_{x} \mathrm{Ru}_{3} \mathrm{Si}_{2}$ in the whole concentration range $x$, making use of our Eq.(40). One sees that the experimental behavior is well reproduced at $\epsilon_{f}^{e f f}=600 \mathrm{~K}$ and $m^{*} / m=1.36$. Small mass ratio is well explained in terms of large band masses for $R u \mathrm{~d}$ electrons [57, 59]. The material provides an example where scattering at small concentrations (on the both sides) plays no significant role on $T_{c}$. That is also in favor of common SC mechanisms (s-wave). 


\section{SUMMARY.}

In conclusion, we have considered phonon (s-wave) superconductivity in the Anderson lattice. Our result indicates that the Kondo effect competes with Cooper pairing, and leads to a dramatic reduction of $T_{c}$. We found that $T_{c}$ may have a re-entrant behavior at somewhat exotic choice of parameters, and that such behavior is stable. We note that our result is similar, but different from the results of Ref. 8] on Kondo alloys, since it does not map on the scattering from paramagnetic impurities [9]. There are no scattering processes in Kondo lattices. Instead, the behavior is coherent. Our results correspond to the low-temperature coherent Fermi-liquid fixed point, which does not map readily on the high-temperature case of free paramagnetic spins. We caution that we consider $\epsilon_{f}^{e f f}$ and $\tilde{V}$ as phenomenological parameters.

In the case of mixed valence, and lower effective mass $m^{*} \sim m_{e}$, we find that $T_{c}$ is substantially less suppressed. Therefore, $T_{c}$ as a function of concentration does not exhibit re-entrant behavior.

On a more qualitative level we would like to add a comment that physics may be different in real systems due to the presence of more than just a single band. It is quite possible that, while one band experiences large mass renormalization via the Anderson hybridization with the f-electrons, the second one does not. At the same time, superconductivity there that is due to a common s-wave mechanism may induce a considerable superconducting gap on the "heavy fermion" band. For instance, in Ref. 60] it was shown theoretically that such a multiband view could even lead to appearance of a non-trivial superconducting order parameter for specific electron bands spectra. Thermodynamics of the superconducting transition reflects the "heaviness" of that first band, while $T_{c}$ itself continues to be due to the mechanisms for the light band. (For results on thermodynamics of multi-band superconductors, see Ref. 61]; these results were recently re-derived by Zhitomirsky and Dao[62]). An example of such a homolog pair may be given by $\mathrm{PrOs}_{4} \mathrm{Sb}_{12}\left(T_{c} \simeq 1.85 K\right)$ and $\mathrm{LaOs}_{4} \mathrm{Sb}_{12}\left(T_{c} \simeq 1 K\right)$ [63].

One of the authors (LPG) acknowledges the partial support from the Alexandr Von Humboldt Foundation and the hospitality of the Max Plank Institute for Chemical Physics, where this work has been initiated. We are grateful for numerous discussions and helpful information to Z. Fisk, P. Gegenwart, C. Geibel, Yu. Grin, M. B. Maple, J. Mydosh, F. Steglich, and H. Wilhelm. 
This work was supported (VB) by TAML at the University of Tennessee and (LPG) by NHFML through the NSF Cooperative agreement No. DMR-008473 and the State of Florida.

[1] K. Andres, J.E. Graebner, and H. R. Ott, Phys. Rev. Lett. 35, 1979 (1975).

[2] A. C. Hewson, "The Kondo problem to heavy fermions", Cambridge University Press (1993).

[3] P. Coleman, C. Pepin, Q. Si, R. Ramazashvili, Journ. of Phys.: Cond. Mat. 13, 723 (2001).

[4] L. P. Gor'kov, Sov. Sci Rev. A. Phys. 9, 1 (1987).

[5] M. Sirgist, K. Ueda, Rev. Mod. Phys. 63, 239 (1991).

[6] J. Bardeen, L. N. Cooper, J. R. Schrieffer, Phys. Rev. 108, 1175 (1958).

[7] A. M. Tsvelick, P. B. Wiegmann, Adv. Phys. 32, 453 (1983).

[8] E. Müller-Hartmann, and J. Zittartz, Phys. Rev. Lett. 26, 428 (1971).

[9] A. A. Abrikosov and L. P. Gor'kov, Sov. Phys. JETP 12, 1243 (1961) [J. Exptl. Theor. Phys. (U.S.S.R.) 39, 1781 (1960)].

[10] G. Riblet and K. Winzer, Solid State Commun., 9, 1663 (1971).

[11] M. B. Maple, W. A. Fertig, C. A. Mota, L.E. DeLong, D. Wohlleben, and R. Fitzgerald, Solid State Commun. 11, 829 (1972).

[12] J. G. Huber, W. A. Fertig, and M. B. Maple, Solid State Commun. 15, 453 (1974).

[13] K. Winzer, Solid State Commun 24, 551 (1977).

[14] R. Dreyer, T. Krug, and K. Winzer, J. Low Temp. Phys. 48, 111 (1982).

[15] P. M. Chaikin and T.W. Mihalisin, Phys. Rev. B 6, 839 (1972).

[16] R. J Delfs, B. J. Beaudry, and D.K. Finnemore, Phys. Rev. B 11, 4212 (1975).

[17] F. D. M. Haldane, Phys. Rev. Lett. 40, 416 (1978).

[18] P. W. Anderson, J. Phys. Chem. Solids 11, 26 (1959).

[19] A. B. Kaiser, J. Phys. C 3, 410 (1970).

[20] H. Shiba, Prog. Theor. Phys. 50, 50 (1973).

[21] P. Schlottmann, Solid State Commun. 21, 663 (1977).

[22] M. B. Maple, L. E. Delong, and B. C. Sales, in "Handbook on the Physics and Chemistry of Rare Earths", eds. by K.A. Gschneider, Jr. and L. Eyring, North Holland, Amsterdam, 1978, p.797. 
[23] T. Matsuura, S. Ichinose, and Y. Nagaoka, Prog. Theor. Phys 57, 1823 (1977).

[24] A. Sakurai, Phys. Rev. B 17, 1195 (1978).

[25] M. Jarrell, Phys. Rev. Lett. 61, 2612 (1988).

[26] E. Schachinger, R. Wunder, Physica 135B, 464 (1985).

[27] M. Azami, T. Matsuura, Physica C 228, 22 (1994).

[28] N. E. Bickers and G.E. Zwicknagl, Phys. Rev. B 36, 6746 (1987).

[29] P. Schlottmann, J. Low Temp. Phys. 47, 27 (1982).

[30] C. R. Proetto and C. A. Balseiro, Phys. Rev. B 31, 2847 (1985).

[31] O. Gunnarsson, K. Schönhammer, Phys. Rev. Lett. 50, 604 (1983); Phys. Rev. B 28, 4315 (1983).

[32] M. Gulácsi and Z. Gulácsi, Solid State Commun. 90, 51 (1994).

[33] U. Rauchschwalbe et. al., Phys. Rev. B 30, R444 (1984).

[34] M. Hakimi and J. G. Huber, Physica 135B, 434 (1985).

[35] F. Steglich, U. Ahlheim, U. Rauchschwalbe, and H. Spille, Physica 148B, 6 (1987).

[36] U. Ahlheim, P. van Aken, H. Spille, and F. Steglich, Helv. Phys. Acta 61, 518 (1988).

[37] J. L. Smith, Z. Fisk, J. O. Willis, A. L. Giorgi, R. B. Roof, H. R. Ott, H. Rudigier, E. Felder, Physica 135B, 3 (1985).

[38] H. R. Ott, H. Rudigier, Z. Fisk, and J. L. Smith, Phys. Rev. B 31, R1651 (1985).

[39] M. B. Maple, J. Alloys and Compounds 303-304, 1 (2000).

[40] U. Rauchschwalbe, F. Steglich, G. R. Stewart, A. L. Giorgi, P. Fulde, K. Maki, Europhys. Lett. 3, 751 (1987); U. Rauchschwalbe, C.D. Bredl, F. Steglich, K. Maki, P. Fulde, ibid., 757 (1987).

[41] Lizardo H. C. M. Nunes, M. S. Figueira, and E. V. L. de Mello, Phys. Rev. B 68, 134511 (2003).

[42] D. M. Newns and N. Read, Adv. Phys. 36, 799 (1987).

[43] P. Coleman, Phys. Rev. B 29, 3035 (1984).

[44] N. Read and D. M. Newns, J. Phys. C 16, 3273 (1983).

[45] S. E. Barnes, J. Phys. F 6, 1375 (1976).

[46] A. J. Millis and P.A. Lee, Phys. Rev. B 35,3394 (1987).

[47] D. M. Edwards, in "Narrow band Phenomena", NATO ASI Series, New York:Plenum, p.23 (1988). 
[48] P. Fulde, in "Narrow band Phenomena", NATO ASI Series, New York:Plenum, p.27 (1988).

[49] A.A. Abrikosov, L. P. Gor'kov, I. E. Dzyaloshinski, "Methods of Quantum Field Theory in Statistical Physics", Dover (1977).

[50] P. Fulde and R. A. Ferrell, Phys. Rev. 135, A550 (1964).

[51] A. I. Larkin and Yu. N. Ovchinnikov, Zh. Eksp. Teor. Fiz. 47, 1136 (1964) [Sov. Phys. JETP 20, $762(1965)]$.

[52] F. Steglich, J. Aarts, C. D. Bredl, W. Lieke, D. Meschede, W. Franz, and H. Schäfer, Phys. Rev. Lett. 43, 1892 (1979).

[53] G. W. Hull, J. H. Wernick, T. H. Geballe, J. V. Waszczak, and J. E. Bernardini, Phys. Rev. B 24, 6715 (1981).

[54] H. Wilhelm and D. Jaccard, Phys. Rev. B 66, 064428 (2002).

[55] K. Maezawa, S. Sakane, T. Fukuhara, H. Ohkuni, R. Settai, Y. Onuki, Physica B 259-261, 1091 (1999).

[56] P. Gegenwart, F. Kromer, M. Lang, G. Sparn, C. Geibel, and F. Steglich, Phys. Rev. Lett. 82, 1293 (1999).

[57] Y. Kishimoto, Yu Kawasaki, and T. Ohno, Physics letters A317, 308 (2003).

[58] U. Rauchschwalbe, U. Ahlheim, U. Gottwick, W. Lieke, and F. Steglich, pp. 231-232, LT17(Contributed Papers), eds U. Eckern, A.Schmid, W. Weber, H. Wühl, Elsevier Science Publisgers B.V. (1984).

[59] Y. Kishimoto, Yu Kawasaki, T. Ohno, T. Hihara, K. Sumiyama, L. C. Gupta, and G. Ghosh, Physica B 329-333, 495 (2003).

[60] D. F. Agterberg, V. Barzykin, and L. P. Gor'kov, Phys. Rev. B 60, 14868 (1999).

[61] B.T. Geilikman, R. O. Zaitsev, and V. Z. Kresin, Sov. Phys. Solid State 9, 642 (1967).

[62] M.E. Zhitomirsky and V.-H. Dao, Phys. Rev. B 69, 054508 (2004).

[63] M. B. Maple, P. C. Ho, N. A. Frederick, V. S. Zapf, W. M. Yuhasz, E. D. Bauer, A. D. Christianson, A. H. Lacerda, J. Phys.:Condens. Matter 15, S2071 (2003). 